\title{
MIBS BREADBOARD READY FOR TESTING
}

\author{
Johan Leijtens $^{(1)}$, Bryan de Goeij ${ }^{(2)}$, Erik Boslooper ${ }^{(3)}$ \\ ${ }^{(1)}$ TNO Science and Industry, Postbus 155, 2600 AD Delft, email: johan.leijtens@.tno.nl \\ ${ }^{(2)}$ TNO Science and Industry, Postbus 155, 2600 AD Delft, email:bryan.degoeij@.tno.nl \\ ${ }^{(3)}$ TNO Science and Industry, Postbus 155, 2600 AD Delft, email: erik.boslooper@.tno.nl
}

\begin{abstract}
MIBS is a spectrometer operating in the thermal infrared wavelength region, designed in frame of the phase A study for the ESA EarthCARE mission as part of the multispectral Imaging instrument MSI, which uses a 2D microbolometer array detector in stead of the more common MCT detectors.

Utilization of a microbolometer and using an integrated calibration system, results in a sensor with a size and mass reduction of at least an order of magnitude when compared to currently flying instruments with similar spectral resolution.

In order to demonstrate feasibility a breadboard has been designed, which will be build and aligned in 2006 and will be ready for testing the forth quarter of 2006.
\end{abstract}

\section{INTRODUCTION}

EarthCARE is a joint European-Japanese mission addressing the need for a better understanding of the interactions between cloud, radiative and aerosol processes that play a role in climate regulation.

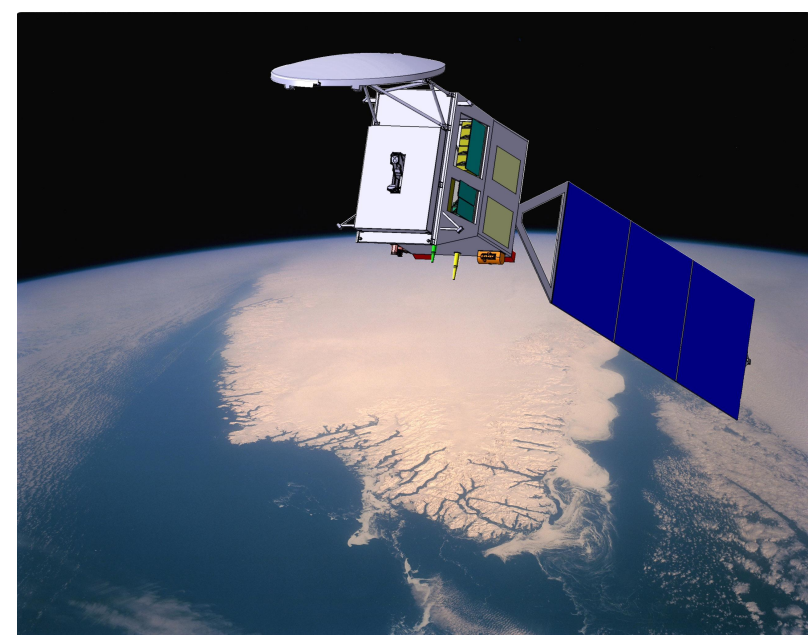

Fig. 1. Earthcare artists impression (ESA Website)

The EarthCARE mission aims to improve the representation and understanding of the Earth's radiative balance in climate and numerical weather forecast models by acquiring vertical profiles of clouds and aerosols, as well as the radiances at the top of the atmosphere.

Earth Observing Systems XI, edited by James J. Butler, Jack Xiong

Proc. of SPIE Vol. 6296, 62961C, (2006) · 0277-786X/06/\$15 - doi: 10.1117/12.674755

Proc. of SPIE Vol. 6296 62961C-1 
The EartCARE mission will fly four instruments, being:

- Backscatter Lidar (ATLID) - ESA High-spectral resolution and depolarisation

- Cloud Profiling Radar (CPR) - JAXA/NICT -36 dBZ sensitivity, 500 m vertical range, Doppler

- Multi-Spectral Imager (MSI) - ESA 7 channels, $150 \mathrm{~km}$ swath, $500 \mathrm{~m}$ pixel

- Broadband Radiometer (BBR) - ESA 2 channels, 3 views (nadir, fore and aft)

Of these the MSI is the only instrument with a swath, thus providing context information.[1]

During the phase A study period there have been two parallel teams studying concepts for the Earthcare satellite.

Within both teams, the MSI instrument is split into different sections:

- Visible Near- and Short wave infrared (VNS)

- Thermal infrared.(TIR)

Due to mass and size restraints, the TIR instruments use microbolometer detectors as the prime sensing elements.

During the phase A study for which an extension is still running, two teams (one lead by Astrium, the other by Alcatel) have performed a preliminary design for both the mission and the instruments.(see technical annex belonging to [1])

TNO Science and Industry (formerly TNO TPD) has taken part in this study as the prime contractor for the Multispectral Imager instrument as part of the Astrium team.

\section{MICROBOLOMETER SPECTROMETER (MIBS)}

Within the Astrium team, TNO has designed a novel concept for the Thermal infrared channels.

Fig. 2. depicts the result of the phase A study as presented in the final report.

The smaller section contains the VNS channels, and the larger section is the so called microbolometer spectrometer.

A number of years ago, this concept couldn't give a high enough NETD in order to be feasible for monitoring of low temperature targets.

However, continuous performance improvements in obtainable noise levels for microbolometers (that are still going on) have led to the availability of bolometer detectors that could fulfill and even exceed the requirements of MSI while using the spectrometer concept.

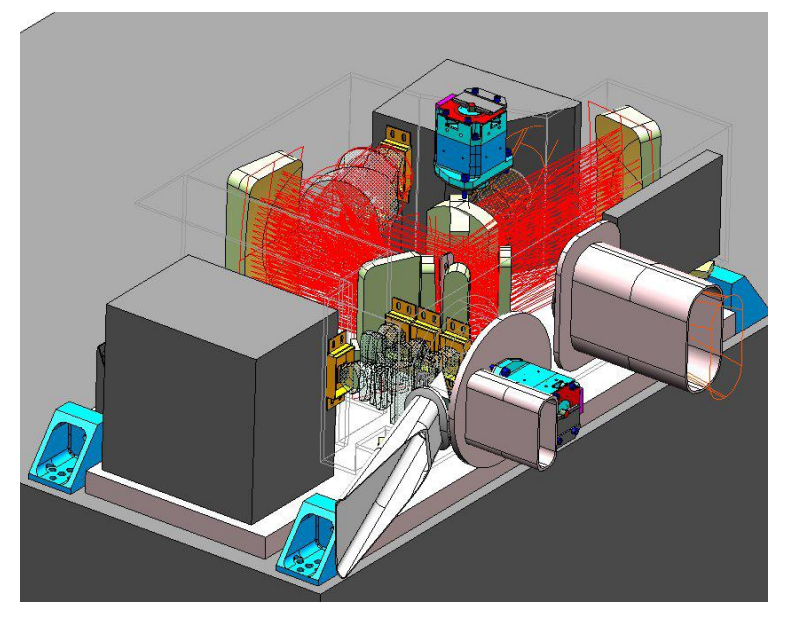

Fig. 2. Phase A MSI instrument 
Because these improvements are mainly driven by military applications within the USA, ITAR restrictions will be a main problem in obtaining these detectors, but due to the fact that microbolometers are also finding their way in things like automotive safety and industrial applications, it is very likely that improved detectors will be available within the foreseeable future from sources outside of the USA.

A number of candidate detector suppliers from outside the USA have been identified that are deemed technologically capable of supplying detectors with sufficient performance.:

- Ulis, France, uses amorphous silicon technology

- $\quad \mathrm{SCD}$, Israel, uses Vanadium oxide (VOx) based bolometers

- INO, Canada, uses both vanadium oxide and Ytrium Barium Cupper Oxide (YBaCuO) based bolometers

- ACREO, Sweden, uses Silicon Germanium quantum well material based bolometers

- NEC,Japan, uses Vanadium oxide based bolometers

Each of these alternative materials have their own merits and drawbacks.

- VOx is a well established material that gives a good quality bolometer which are currently mass produced.

- Amorphous silicon is cheap to manufacture but has a relatively high noise figure

- $\mathrm{YBaCuO}$ potentially gives a higher performance [2] but is less mature then VOx.

- $\quad$ SiGe quantum well material has a very low noise and good response, but is also new.[4]

Noise performance of bolometer detectors has improved by more than a factor of three in the last four years and there is no indication that performance cannot be improved any further. Main developments are focused on a number of issues:

- Improved noise performance (NETD)

- Smaller pixel sizes

- Increased temporal response

- Cheaper packaging

- Higher yield

For the MSI, the improvement of the NETD is the most important one closely followed by the reduction in pixel size (as this will allow us to decrease the size of the optical bench).

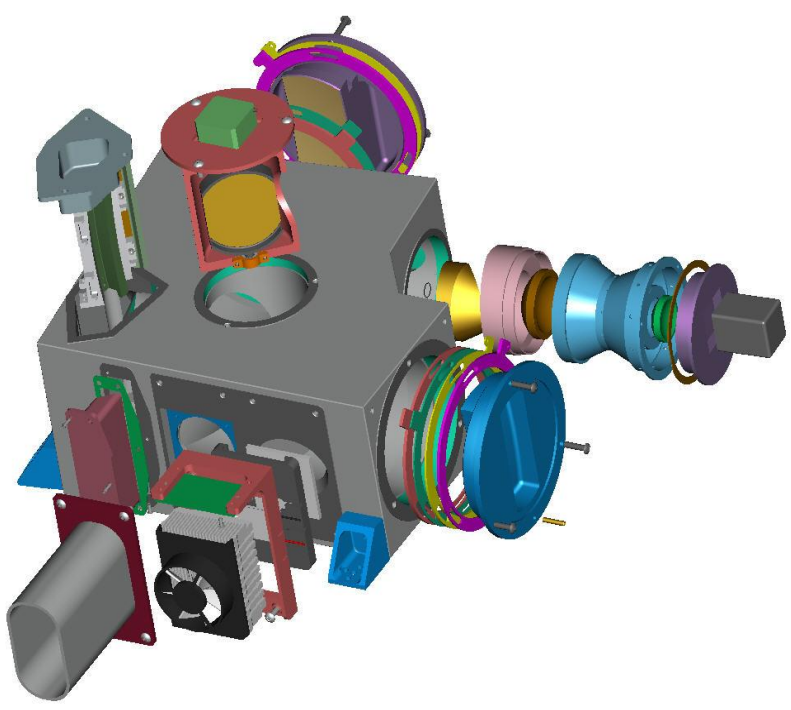

Fig. 3. Mechanical setup MIBS 
Although spectrometers seem to have a radiometric disadvantage as compared to the more conventional beamsplitter operated systems (which is disputable for instruments with more than 2 channels due to the additional absorption in the needed beamsplitters) the main advantage is the higher spectral purity and the larger number of channels that can be provided at the same or marginally increased costs. (Some extra signal processing electronics might be needed for the instrument control unit, and at satellite level more data will need to be handled.)

Despite extensive modeling that has been performed which proves the feasibility of the concept, it was decided to build a breadboard to actually prove the feasibility of the concept through real measurements. The breadboard will closely resemble the final instrument, apart from the facts that it will not be light weighted and will operate at room temperature. The main assemblies (slit assembly and camera assembly) as well as the mirrors are all highly similar to the foreseen flight configuration. The breadboard therefore, can easily be converted into a flight capable instrument through exchange of the pointing mirror assembly, the cold blackbody and the detector. (If necessary the optical bench can be light weighted also)

Figure 3 depicts the current configuration of the breadboard as it is currently being manufactured. The optical bench is a monolithic structure that is used to keep the temperature of the entire setup as constant as possible. Being temperaturebalancing devices instead of photon detectors, microbolometers are not very sensitive to the absolute temperature of operation (which for photon detectors is largely influencing the noise behavior) but are more sensitive to small temperature variations in case absolute temperature measurements are to be performed. Therefore instead of a cryogenic cooler, a high stability thermal control loop is needed. This partially offsets the power advance that can be obtained by leaving out the cooler. Nevertheless the power consumption of an instrument using microbolometers can be significantly less than that of an actively cooled instrument. In any event the lack of mechanical vibrations is a main advantage for use on board of a satellite.

From the conceptual point of view bolometer instruments can have another advantage over photon detectors in case a spectrometer is used. Photon detectors exhibit a long wavelength cutoff that is related to the bandgap voltage of the detectors, but microbolometers are inherently broadband devices. The necessary limitation of the wavelength response is mostly due to the fact that a certain temporal response is required and therefore the therefore limited absorber thermal mass. For instruments like GERB some very broadband devices have been produced (http://gerb.oma.be/gerb/Mission/introduction.html), which can only have a limited temporal resolution but are nevertheless very sensible for measuring the overall radiation coming from the earth.

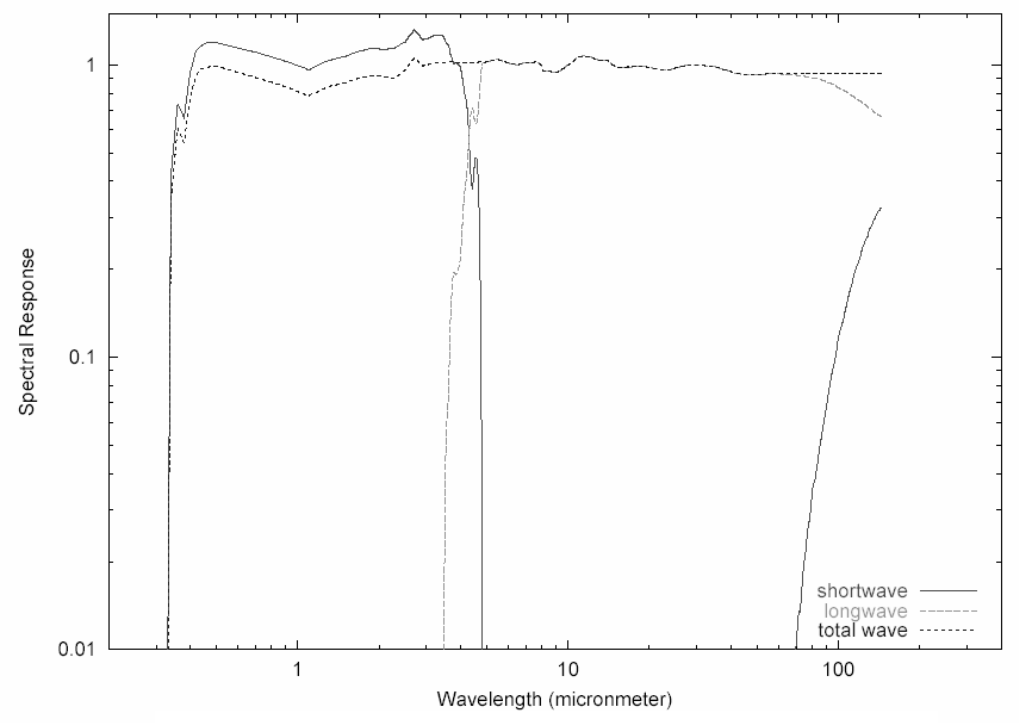

Fig. 4. GERB spectral response (courtesy RMI Belgium) 
In an attempt to increase the temporal response but maintain the broadband response, attempts have been taken to use multilevel mirrors and novel absorber materials.[3] but until now, no microbolometers have been designed that have a very broadband response in combination with a temporal response that would allow real-time 2D imaging. When looking at figure 5, it can be deduced that for earth observation applications this may not be needed.

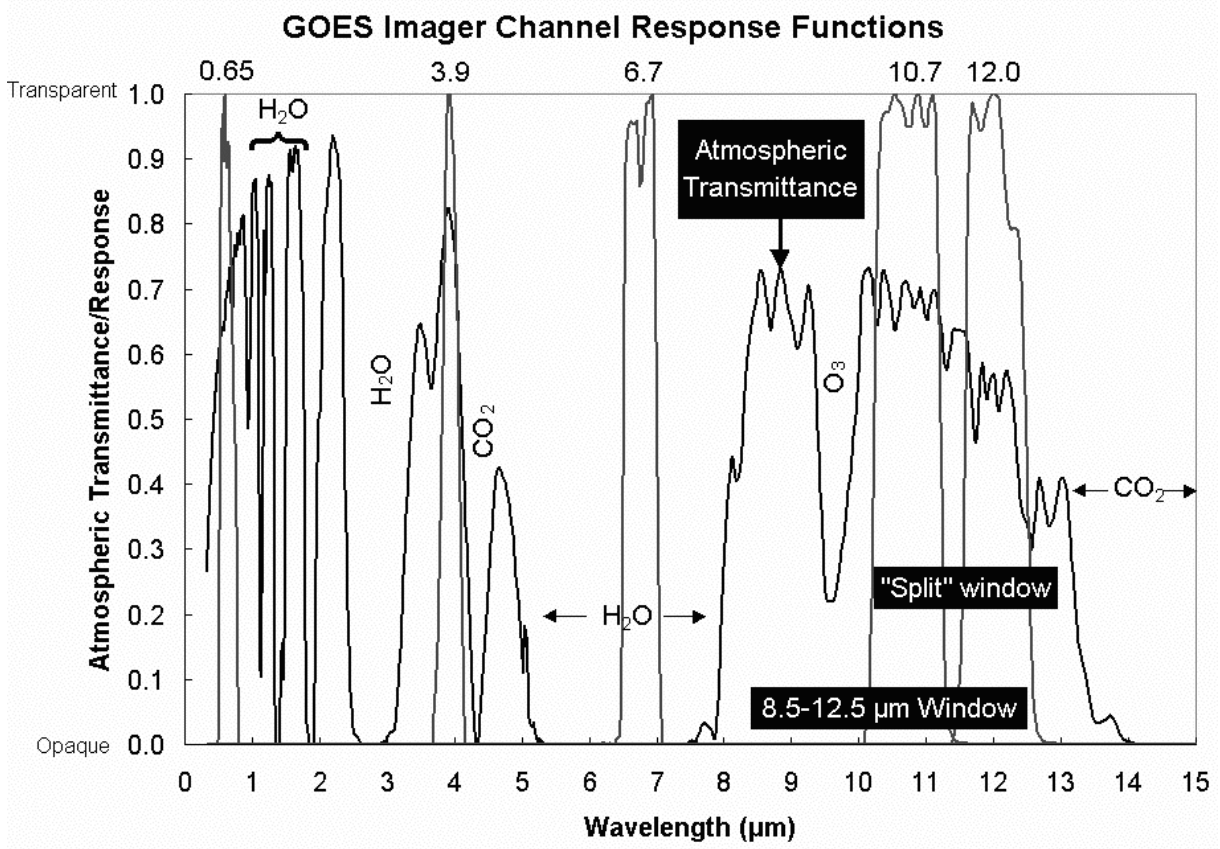

Fig. 5. GOES and atmospheric transmission profiles

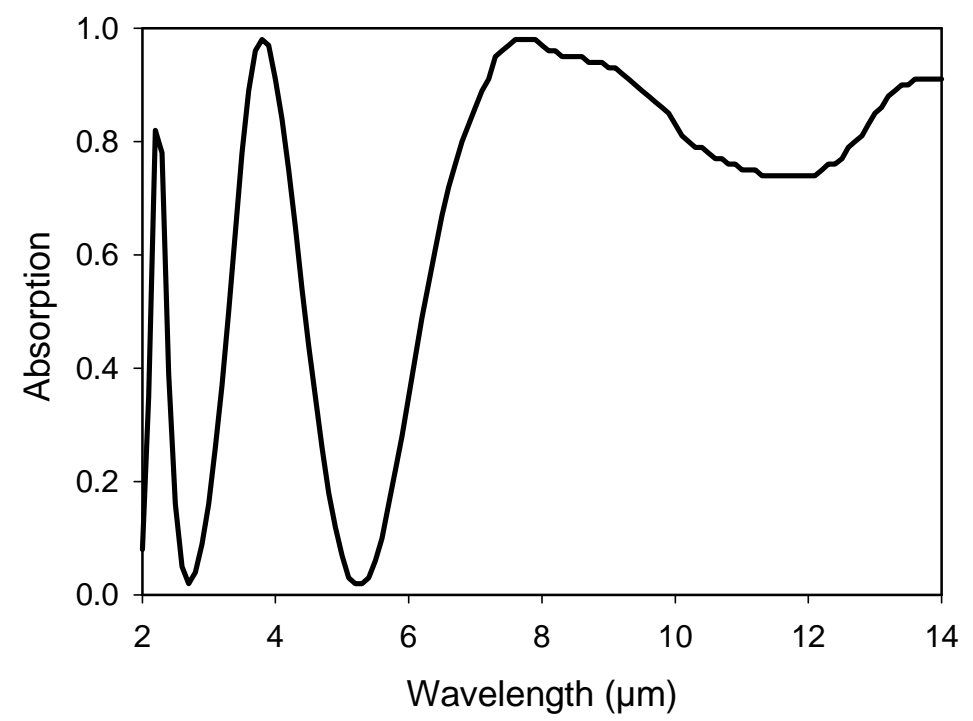

Fig. 6. multi band absorption profile

As can be seen above, the atmosphere is only transparent for certain wavelengths, the so-called atmospheric windows. Therefore only specific wavelengths are needed since additional wavelengths will not give you any additional information. By designing a microbolometer that has a significant response in both the 3 to 5 and 8 to 12 micron window, both main atmospheric windows can be covered while retaining a fast temporal response. The feasibility of this 
type of absorption has been proven not only by design, but also by production and measurement. A designed pixel response is given in Fig 6. Using this response (and an instrument like MIBS which is capable of imaging both wavelength regions at the same time) most probably a number of significant measurements can be performed simultaneously:

- Surface temperature for room ambient temperature targets (split window 10.7 and 12 micron bands)

- Surface temperature for high temperature targets (in $3 . .5$ micron band)

- $\mathrm{H} 2 \mathrm{O}$

- $\mathrm{CO} 2$

- $\mathrm{O} 3$

The first three measurements can be performed for sure, the last two very likely but this has never been proven before. Especially the $\mathrm{CO} 2$ measurements would be of significant importance because $\mathrm{CO} 2$ and water are recognized as the two main greenhouse gasses.

The ability to distinct between low and high temperature targets will allow you to monitor environmental disasters like forest fires and volcanic eruptions, which is not possible in the $8 . .12$ micron band only because without additional information it is impossible to distinct between sunglint /urban heat and a high temperature events. The high dynamic range of input signals would require a very high-resolution analog to digital conversion and associated input range or the resolution for low temperature targets would be impaired.

Due to the fact that the spectrometer uses reflective optics for the largest part possible and only a very limited amount of $\mathrm{ZnSe}$ and Ge for the lenses and the prism, the inherent limitations as for the wavelength response are currently set by the germanium. The transmission below 2 micron is very low for Germanium, which sets a lower limit and absorption significantly increases for wavelengths larger then 13 to 14 micron (which may prove to be the limiting factor for $\mathrm{CO} 2$ detection).

\section{MANUFACTURING STATUS}

Currently the parts to be used for the breadboard have been ordered and are under production. The current planning calls for completion of the production by the end of June and is more or less in line with the previously foreseen schedule. Assembly will be performed in July/early August if everything follows the current planning, which has led to the title of this paper (ready for testing). Although at this time (during the writing of the paper) the breadboard is not ready for testing yet, there is good hope that at the time of the conference (second half of august) the assembled breadboard will be ready for testing.

As can be seen in the following pictures, especially the optical parts are already nearing completion.

It is the intention of TNO to assemble the breadboard before the end of the year and do some first order characterization measurements.

The aim of this project (which is supported by the Dutch NIVR) is to show that the final instrument can meet the requirements as posed on the EarthCARE MSI instrument once the foreseen detectors are available.

\section{CONCLUSION AND ACKNOWLEDGEMENT}

The production of parts for MIBS is on the way and at schedule.

We would like to thank NIVR for their support to this program, allowing the demonstration of what may be a new breed of thermal infrared instruments. 


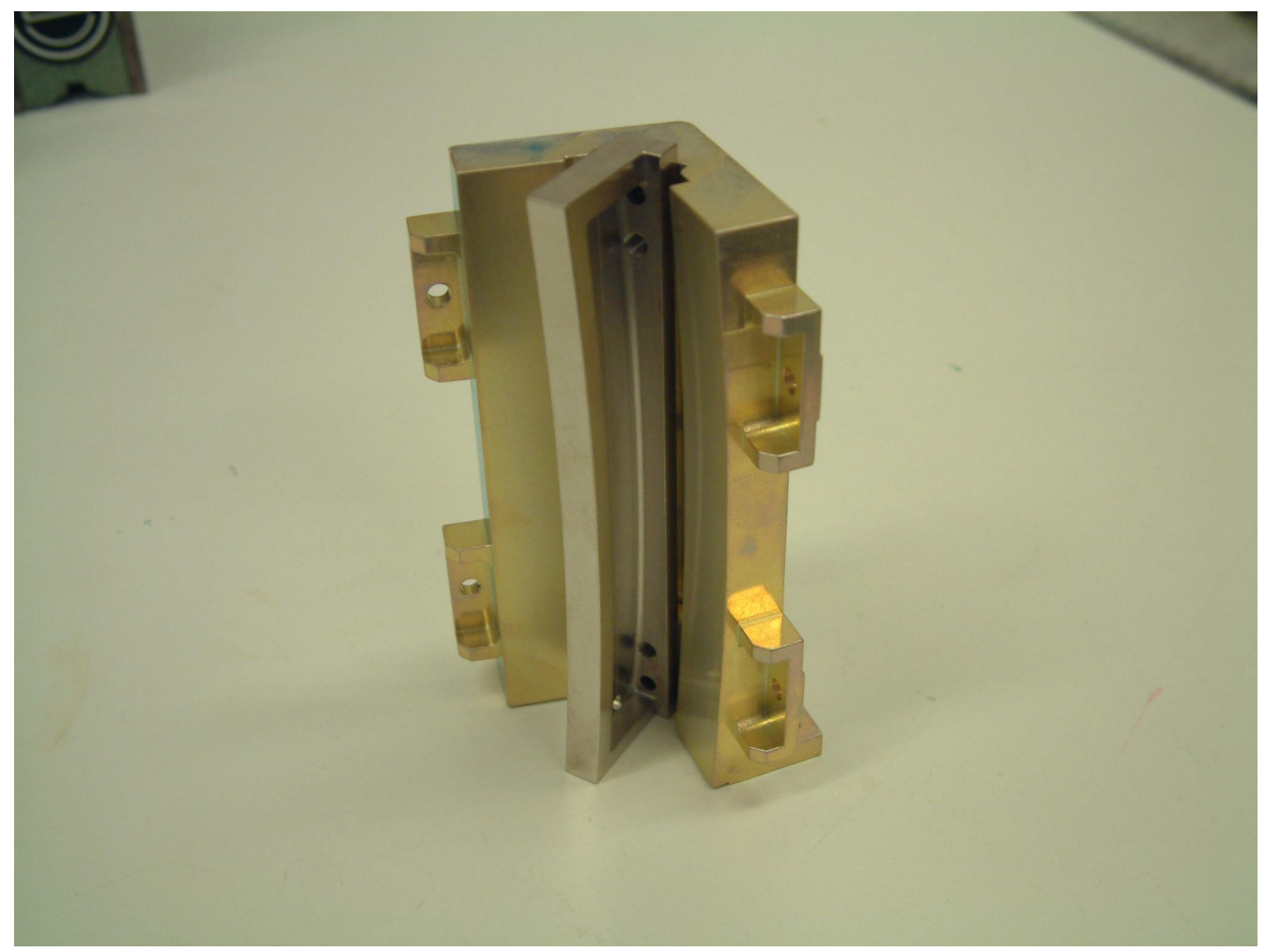

Fig. 7. Slit assembly central part

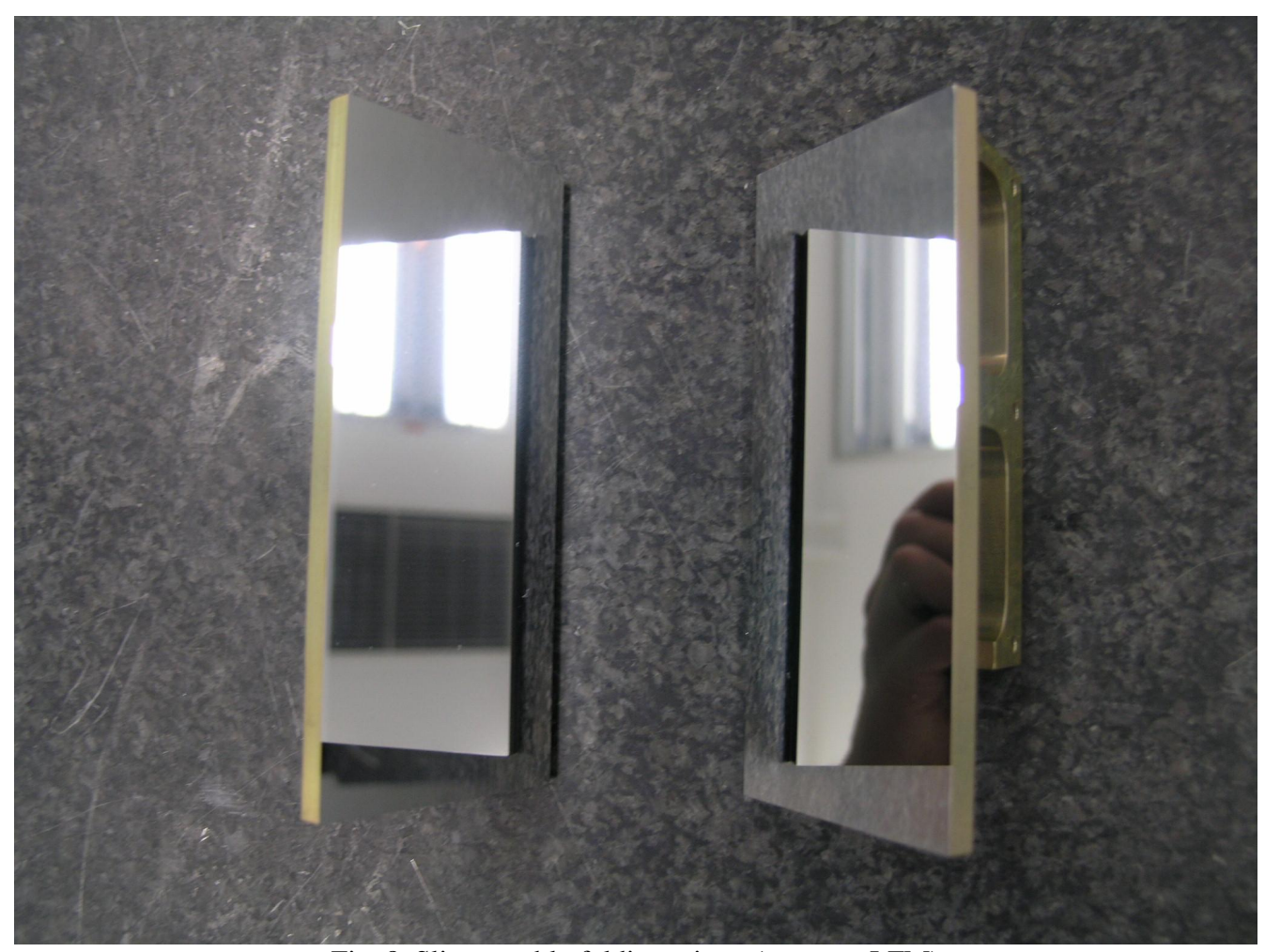

Fig. 8. Slit assembly folding mirror (courtesy LFM)

Proc. of SPIE Vol. 6296 62961C-7 


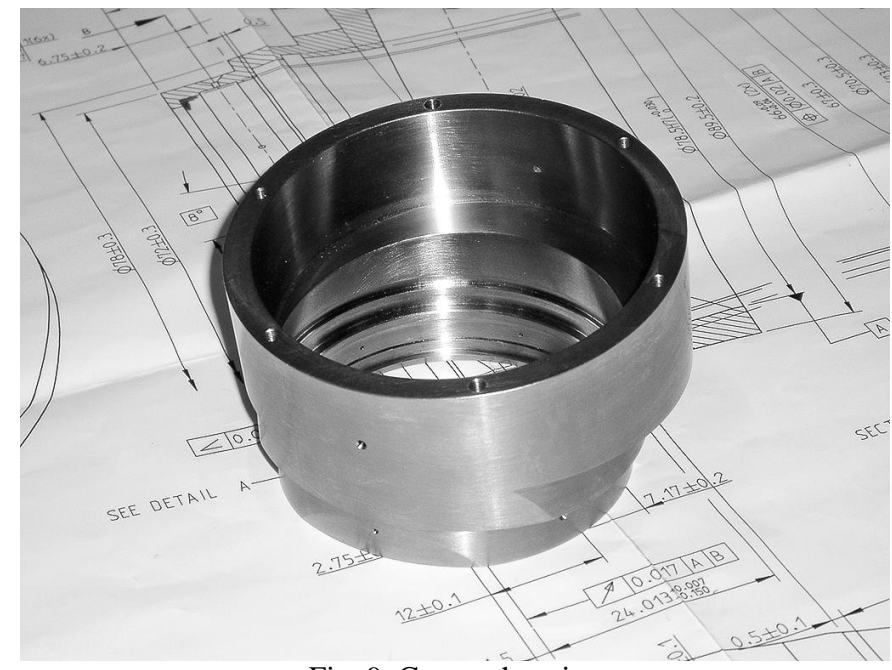

Fig. 9. Camera housing

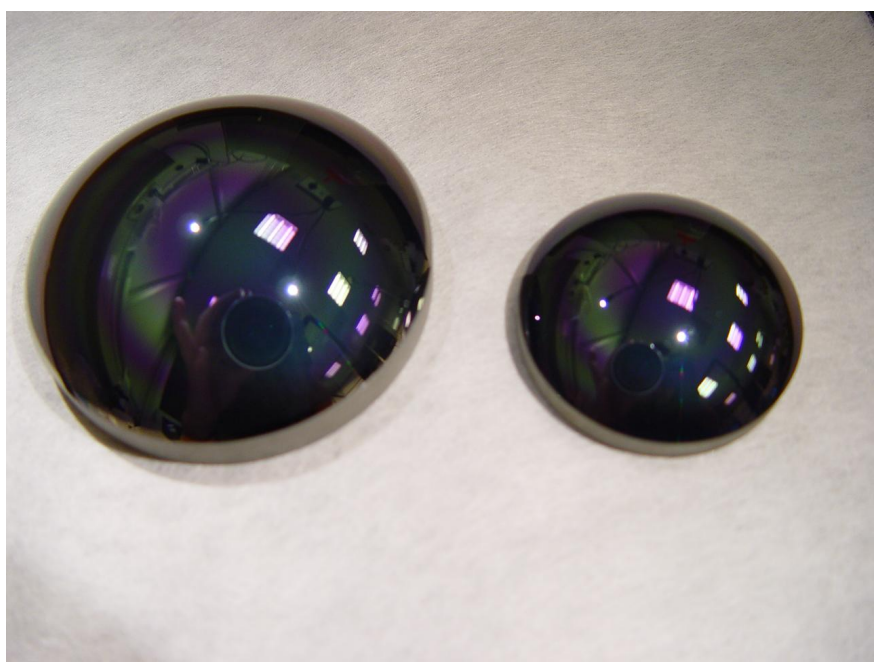

Fig. 10. Camera lenses

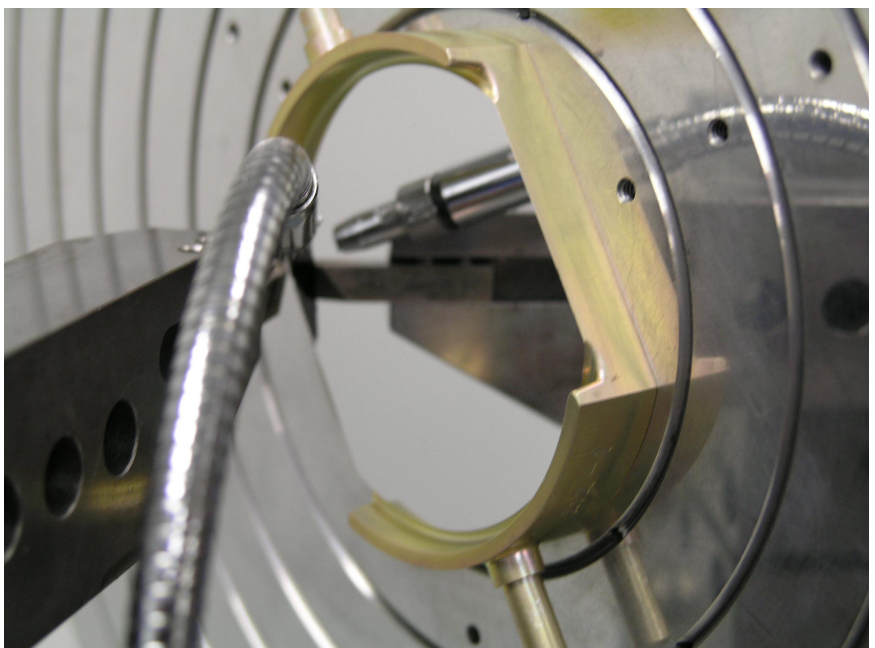

Fig. 11. Pointing mirror during single point diamond turning (courtesy LFM)

Proc. of SPIE Vol. 6296 62961C-8 


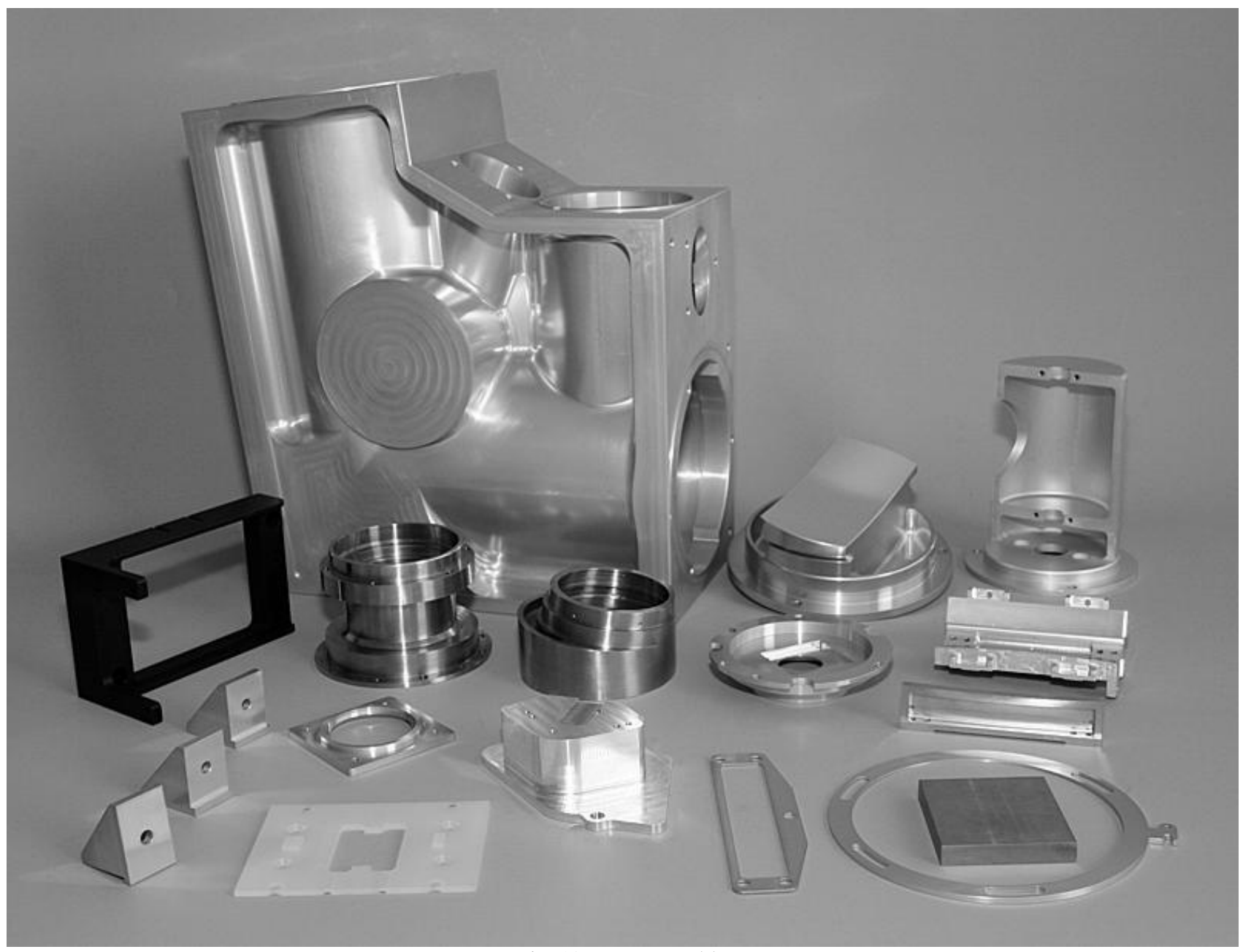

Fig. 12. parts assembly

\section{References}

[1] Earthcare, Earth, Clouds, Aerosols and radiation explorer ESA report SP 1257(1) and technical and programmatic annex

[2] L. Ngo Phong and S.N. Qiu, Room temperature YbaCuO microbolometers., Journal Vac. Science Techn. A 18(2), Mar/Apr. 2000

[3] Donald Butler, Broadband Visible 0.3 through $100 \mu \mathrm{m}$ YBaCuO Uncooled MicrobolometersProject \# 575-95

[4] S. G. E. Wissmar, et all High signal-to-noise ratio quantum well bolometer materials, SPIE [6401-23] 